\title{
Application of the complex network method in solid-state sintering
}

\author{
Chao Wang, Shaohua Chen* \\ LNM, Institute of Mechanics, Chinese Academy of Sciences, Beijing 100190, China
}

\section{A R T I C L E I N F O}

\section{Article history:}

Received 23 September 2012

Received in revised form 8 November 2012

Accepted 19 November 2012

Available online 23 December 2012

\section{Keywords:}

Solid-state sintering

Complex network

Discrete element method

Force chain

Microstructure evolution

\begin{abstract}
A B S T R A C T
The microstructure evolution and force distribution during solid-state sintering are studied at the particle length scale for a planar layer system of copper particles. The complex network as well as discrete element methods (DEM) is adopted, in which the contact network and the force network are mainly considered. Comparing the contact network to the microstructure evolution, we find that pores always form in a large contact polygon. In the force network, the normal contact forces distribute extremely non-uniform. An interesting phenomenon is that a ring-like structure of force chain emerges spontaneously in the sintering force networks, whose life expectance is much longer than other linear force chains. Furthermore, a high contact force is more likely to form at a new contact site. To our knowledge, it is the first time to study force distributions and microstructure evolutions in sintering from the complex network point of view, which should be a promising method for further investigating the complex sintering process in the future.
\end{abstract}

(c) 2012 Elsevier B.V. All rights reserved.

\section{Introduction}

Solid-state sintering is a thermal treatment process of ceramic or alloy granular compacts at a high temperature to achieve desired mechanical and material properties [1]. In granular compacts, particles contact and interact with their neighbors and all contacts among neighboring particles constitute a complex contact network. During sintering, contact particles in compacts fuse with each other by a special force with the underlying mechanisms of grain boundary diffusion and surface diffusion [2]. Interacting forces are transmitted through the inter-particle contacts and the magnitudes vary significantly at different regions due to the different contact configurations, which lead to a complex force network accompanying with the contact network. During the period of sintering, some contact interfaces break accompanying new forming contacts $[3,4]$. As a result, both the contact network and the force network in sintering compacts would vary with the sintering process. To characterize the topology and evolution characteristics of the two complex networks as well as unveil their correlations is an important issue, which should give a much better understanding of practical sintering.

However, it is very difficult to observe the sintering process and disclose the microstructure evolution experimentally due to a high temperature. Numerical experiments are indispensable choices for researchers, such as finite element method (FEM) and discrete element method (DEM). Since the initial state of a sintering system is discrete, DEM should be a better technique. In fact, DEM is a gen-

\footnotetext{
* Corresponding author. Tel.: +86 10 82543960; fax: +86 1082543977 .

E-mail address: chenshaohua72@hotmail.com (S. Chen).
}

eral simulation scheme that is originally introduced in the study of rock mechanics [5]. In DEM, each particle is modeled as a sphere that interacts with its neighbors through an appropriate force law and its motion depends on the interactions with all the neighboring particles. In contrast to continuum mechanics models [6,7], DEM takes the granular nature of materials into account and allows the investigation of phenomena at the particle length scale, such as particle rearrangement [8,9] and agglomeration [10,11]. It also allows access to mesoscopic properties, such as position, velocity, coordination number of each particle. In recent years, DEM has also been successfully applied to investigate particle sintering, such as densification rates $[8,9,12]$, viscosities [8,13], anisotropic sintering [14], constrained sintering [15] and the evolution of cracks during sintering $[8,16]$.

Based on the capability of accurately determining each particle's position and the interaction force at any moment, DEM should be able to give useful insights on the topology and microstructure evolution of a sintering system through establishing a complex network among particles, such as the contact network and force network. Lots of researchers have already realized that a particlesintering system can be viewed as a complex network [13]. However, the complex network method is not applied to study the particle sintering problem so far.

As a very useful technique, the theory of complex network has been well developed in the fields of anthropology, psychology, communication studies, information science, organizational studies, economics, biology and neuroscience [17-21]. It is a mathematical model to characterize the complex relations among a class of groups. Numerous groups in the real world could be well-described by a network structure, such as a network of 
websites, neurons and people. A complex network can be described as a set of edges that connect different nodes. In different systems, edges and nodes in the corresponding networks have different meanings. For example, in a granular system, nodes and edges represent particles and the physical contacts among them, respectively.

Specially, in a granular system, the technique allows a better description of the anisotropic characteristics of contact topology and contact force, which could give a clear picture of the evolution and correlation of the two complex networks [22-32], but all the studied granular compacts belong to non-sintering systems. The main difference between the sintering and non-sintering systems is the interacting force among particles. Is the complex network method useful for understanding of particle sintering process?

Researchers are accustomed to characterizing the particle sintering system from the pore point of view [33]. A series of parameters related to pores, such as pore size, pore shape, distribution of pore size, are measured experimentally using different techniques [1], such as optical microscopy, electron microscopy and mercury porosimetry. All these parameters have been introduced theoretically in the constitutive relations of sintering systems [6]. For the sake of simplicity and quantitative description, pores are assumed to be closed, isolated and spherical [34]. However, experiments found that such kinds of pores only emerge at the second stage of sintering, i.e., the relative density larger than 90\% [35]. At the initial stage of sintering, pores are very complex, such as irregular shape, interconnection and even open to the compact surfaces. Actually, pores in a particle-sintering system could also be viewed as a structure built by particles. Is it possible to investigate the evolution of pores from the particle point of view using the complex network method?

Furthermore, anisotropic force in particle-sintering systems is usually described as stress tensors in the continuum sintering theory [6,7]. It is a statistical and average concept and can neither give us a clear picture of force at the particle length scale nor help us to understand the phenomena happening at this scale, such as particle rearrangement and agglomeration. Inspired by the interesting force network in a non-sintering granular media [24], we expect to find some new phenomena of force networks in particle-sintering systems.

Two main problems will be studied in the present paper using the discrete element method and the complex network. One is the evolution characteristic of pores and the other is features of contact force networks in sintering. The remainder of this paper is organized as follows. In Section 2, the simulation model studied in the present paper is given and the special interaction forces in sintering are briefly introduced. The feasibility of DEM applied to the force network of a sintering-system is studied in Section 3. Simulation results are discussed in Section 4, where the relations among the contact network, force network and the micro-structure evolution are mainly focused on. Finally, remarks are given in Section 5 .

\section{Numerical model and the interaction forces in sintering}

A two-dimensional sintering model of a planar layer of copper particles is investigated in the present paper. The model consists of 500 uniform copper spheres with radius $127 \mu \mathrm{m}$ and the particles distribute randomly in a circular region with diameter $6.35 \mathrm{~mm}$. The sintering temperature is set as $1300 \mathrm{~K}$ and the tangential viscosity $\eta$ takes 0.001 in our simulations. The other sintering or material parameters are given in Table 1.

Discrete element method (DEM) combining with complex network will be used to simulate the sintering process. Copper particles in the sintering system are treated as discrete elements, which
Table 1

Parameters used in the numerical simulation for copper sintering (adopted from [12]).

\begin{tabular}{llll}
\hline$\delta_{\mathrm{b}} D_{0 \mathrm{~b}}\left(\mathrm{~m}^{3} / \mathrm{s}\right)$ & $\mathrm{Q}_{\mathrm{b}}(\mathrm{kJ} / \mathrm{mol})$ & $\gamma\left(\mathrm{J} / \mathrm{m}^{2}\right)$ & $\Omega\left(\mathrm{m}^{3}\right)$ \\
\hline $5.12 \times 10^{-15}$ & 105 & 1.72 & $1.18 \times 10^{-29}$
\end{tabular}

interact with each other through the sintering force $[9,12,15,16]$. The normal component $N_{\mathrm{s}}$ and the tangential one $T_{\mathrm{s}}$ of the contact force at the contact interface can be expressed as:

$N_{\mathrm{s}}=\frac{\pi a_{\mathrm{s}}^{4}}{8 \Delta_{\mathrm{b}}} \frac{d h}{d t}-\frac{9}{8} \pi R_{p} \gamma_{\mathrm{s}}$

$T_{\mathrm{s}}=-\eta \frac{\pi a_{\mathrm{s}}^{2} R_{p}^{2}}{8 \Delta_{b}} \frac{d u}{d t}$

$\Delta_{\mathrm{b}}=\frac{\Omega}{k T} \delta_{\mathrm{b}} D_{\mathrm{b}}$

where $D_{\mathrm{b}}=D_{0 \mathrm{~b}} \exp \left(-Q_{\mathrm{b}} / R T\right)$ is the diffusion coefficient at the grain boundary with thickness $\delta_{\mathrm{b}}$ and activation energy $Q_{\mathrm{b}}, \Omega$ is the atomic volume, $k$ the Boltzmann constant, $T$ the temperature, $h$ the indentation depth between two spherical particles, $\gamma_{s}$ the surface energy, $R_{p}$ the radius of the particle, $a_{\mathrm{s}}$ the sintering contact radius which grows up according to the Coble's model $a_{\mathrm{s}}=\sqrt{2 h R_{p}}, d_{\mathrm{u}} / d_{\mathrm{t}}$ the tangential component of the relative velocity at the contact site and $\eta$ denotes the viscosity.

At each time step, the resultant force of each particle is calculated to calculate its acceleration. Then, a new velocity and position of the particle can be found and updated.

The mass transport mechanism assumed in Eq. (1) consists of grain boundary diffusion and surface diffusion. The first term on the right hand side of Eq. (1a) denotes the normal viscous force resisting the relative normal motion of two adjacent particles, which can also be found in $[8,14,36]$. The second term denotes the sintering force trying to pull two adjacent particles together. The tangential force $T_{\mathrm{s}}$ in Eq. (1b) has an opposite direction to the tangential component of the relative velocity of each contact particle.

\section{The feasibility of complex network method}

In order to check the influence of time step $\Delta$ on the simulation results, we establish two small sintering systems with different distributions of particles as benchmarks, both of which are circular and consist of 50 uniform copper particles of radius $40 \mu \mathrm{m}$ as shown in Fig. 1a and e, respectively. The diameter of the circular region for calculation is $640 \mu \mathrm{m}$. In Fig. 1a, particles distribute randomly, while regular distribution is regulated in Fig. 1e. The time step $\Delta$ is set as $1 \mathrm{~s}, 0.1 \mathrm{~s}$ and $0.01 \mathrm{~s}$, respectively, for each sintering system. Other simulation parameters are the same as above.

Force network consists of numerous lines that bridge the centers of each pair of contact particles. The red line and the black one represent compressive (attractive) and tensile (repulsive) forces, respectively. The width of each line represents the magnitude of the normal contact force normalized by the average one of all the contact forces in the system at a certain simulation time step. The thicker the network line, the larger the normal contact force is. The characteristics of force networks are mainly focused, especially, the site emerging high contact forces.

Fig. 1b-d display the snapshots of force distributions in the sintering system shown in Fig. 1a, each of which corresponds to 98, 980 and 9800 time steps, respectively. Comparing Fig. 1b, c and $d$, one can see that the high force chains appear not only at the same site but also at the same physical time $98 \mathrm{~s}$. It proves that 

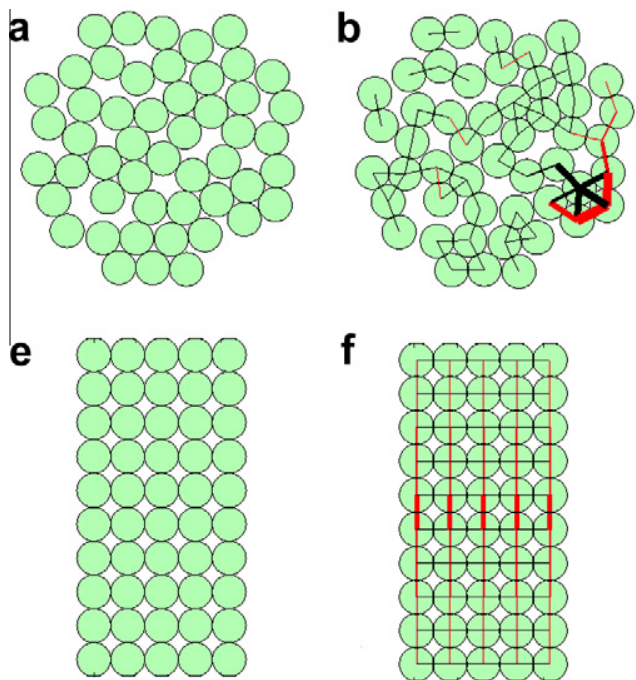

f

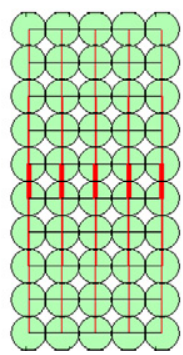

c

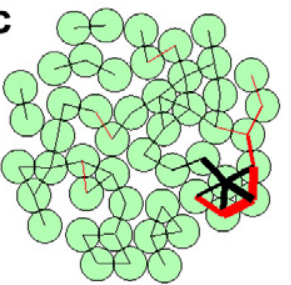

g

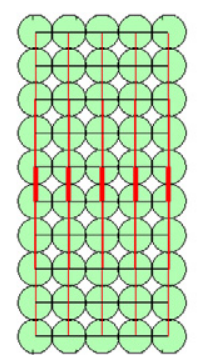

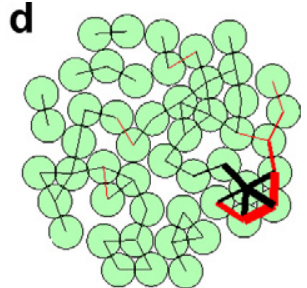

h

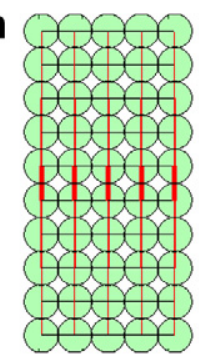

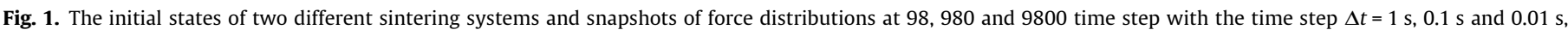

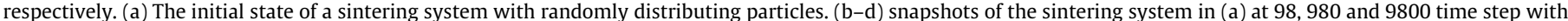

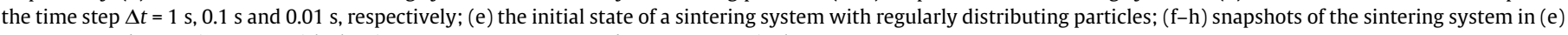
at 98,980 and 9800 time step with the time step $\Delta t=1 \mathrm{~s}, 0.1 \mathrm{~s}$ and $0.01 \mathrm{~s}$, respectively.

the simulation results of force network will not be influenced by the choice of time step. As for the sintering system with a regulated distribution of particles shown in Fig. 1e, the same conclusion could be made from the comparisons of Fig. $1 \mathrm{f}-\mathrm{h}$, each of which corresponds to the snapshot of force distributions at 98, 980 and 9800 time steps, respectively.

In a free sintering process, sintering particles are usually driven by interactions with their neighboring particles and then tune their positions to a more force-balanced state. It is well known that such a force-balanced state is much easier to reach and the contact forces should distribute more uniformly in a regular system than in a random one, which is also evidenced in our simulations shown in Fig. 2. The time step $\Delta$ is set as $1 \mathrm{~s}$ here. Fig. $2 \mathrm{a}-\mathrm{d}$ show the force distributions in the system with randomly distributing particles at 0, 700, 5000 and 10000 time step, respectively. High force chains could be observed at any moment after the initial state. However, in the system with regularly distributing particles, as shown in Fig. 2e-h, high force chains only appear at an intermediate moment and will disappear afterward. Therefore, the existence of high force chains is a basic signal that demonstrates the uniformity of the particle system. The other characteristics of a force network will be further discussed in the following text.

\section{Simulation results and discussions}

\subsection{Contact networks in solid-state sintering}

Contact network consists of lines that connect the centers of all contact particles. An initial state of the contact network is shown in Fig. 3a for the above mentioned simulation model in Section 2. The inter-particle tangential viscosity is $\eta=0$. For comparison, an intermediate state at $5 \times 10^{6}$ sintering time step is shown in Fig. $3 \mathrm{~b}$. From Fig. 3a and b, one can see that the contact network is very complex, including different network structures, such as a single contact line, a contact triangle and contact polygons. A single contact line denotes an isolated pair of particles as shown by an arrow in Fig. 3a. The contact triangle is the closest configuration of contact particles. The region with a high density of contact triangles, a

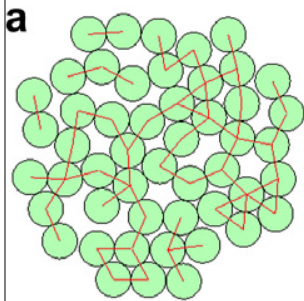

e

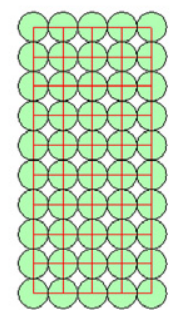

b

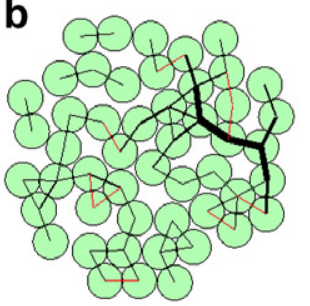

f

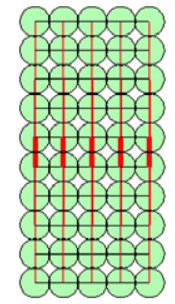

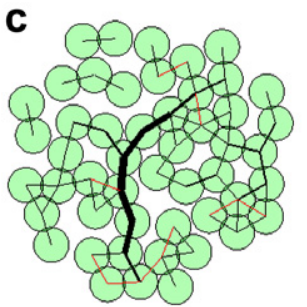

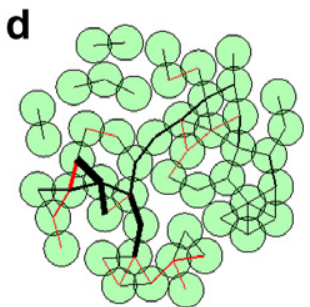

g

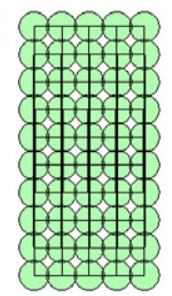

h

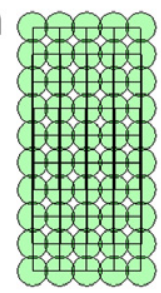

Fig. 2. Snapshots of force distributions of two kinds of sintering systems at $0,700,5000$ and 10,000 time step with $\Delta t=1 \mathrm{~s}$. (a-d) system with randomly distributing particles; (e-h) system with regularly distributing particles. 

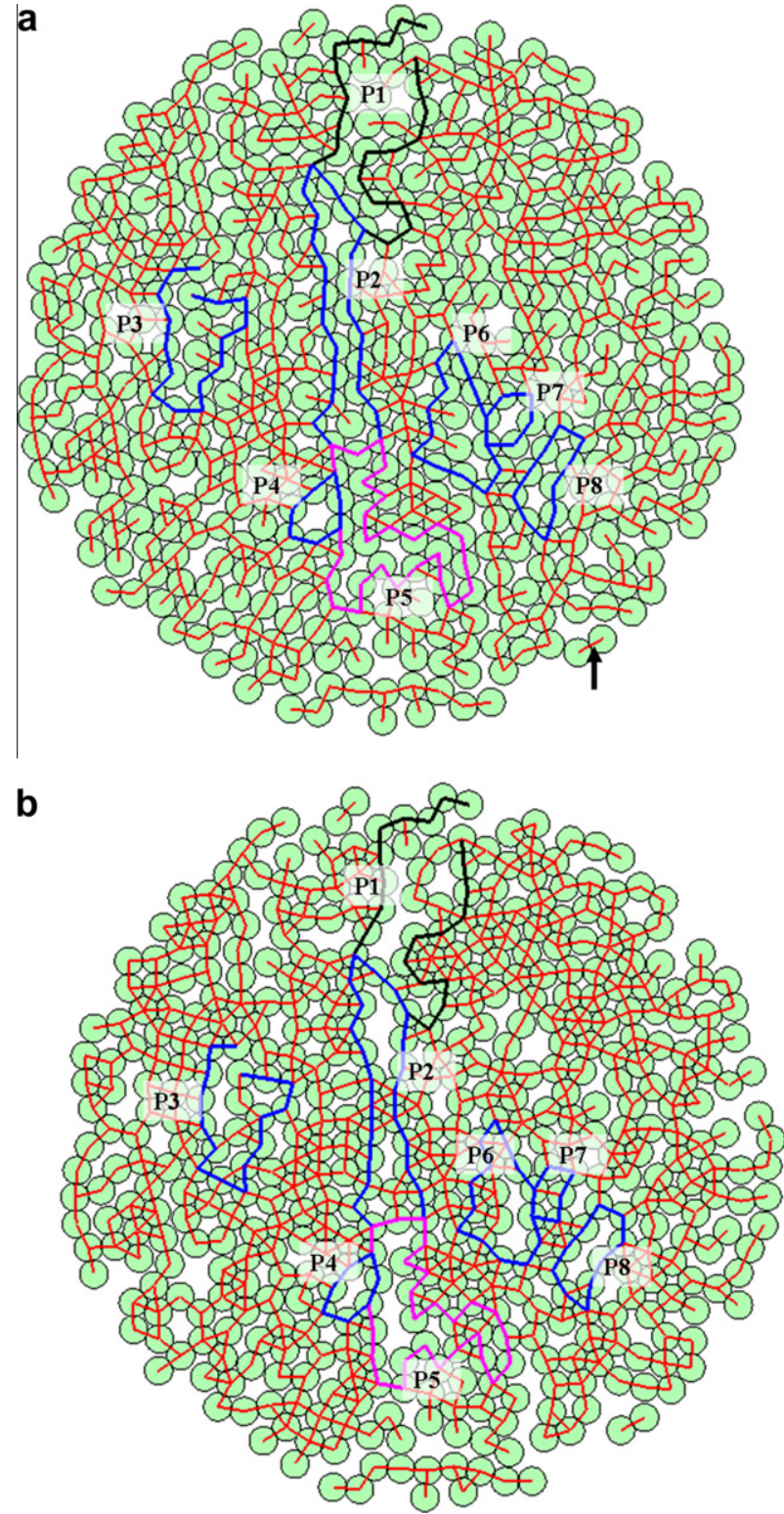

Fig. 3. The contact networks of a particle system containing 500 copper particles of radius of $127 \mu \mathrm{m}$. Eight contact polygons denoted by P1-P8 are highlighted by different colors. (a) the initial state; (b) the state at $5 \times 10^{6}$ sintering time step (For interpretation of the references to colour in this figure legend, the reader is referred to the web version of this article.).

i.e. 3-cycle, is a relatively close and densified one. In contrast, the region encircled by a contact polygon has a poor connectivity. In order to find the relation of contact network and the densification process of the sintering system, eight contact polygons are tagged as P1-P8 in Fig. 3a, which are highlighted with different colors. In the initial state shown in Fig. 3a, some contact polygons are close, such as P2, P4, P5, P6, P7 and P8, while the others are open, such as P1 and P3. Note that all these eight contact polygons, no matter close or open, do not contain any other small contact polygon in them at the initial state. In the sintering process, the sintering system will become denser and denser. We find that new contacts will form in some polygons and the initially large polygons will be divided into several small ones. For example, there are eight new contacts emerging in P2 after sintering, which divide P2 into four small contact polygons and four contact triangles. Similar phenomena also happen in P1, P3, P5, P6 and P7. Large contact polygons collapse into small ones, which results in an increasing number of small polygons and triangles and a decreasing number of initially large contact polygons. Updated density of the sintering system can be measured. An interesting phenomenon is that pores are more likely generated in an initially large contact polygon, while the initially small one tends to annihilate finally. Comparing Fig. $3 a$ and $b$, one can see that, at $5 \times 10^{6}$ sintering time step, pores do form in the relatively large polygons P1, P2, P3, P5 and P6, while the small polygons P4 and P7 experience shrink and finally disappear. All the results suggest that complex contact network should be useful for predicting positions that pores will emerge.

\subsection{Force networks in solid-state sintering}

Force networks have different characteristics at different sintering time. Fig. $4 \mathrm{a}$ and $\mathrm{b}$ show the snapshots of force networks at 5000 and 25,000 simulation time step, respectively. Comparing the two figures, one can see that most of the inter-particle compressive forces at 5000 time step changes to be tensile ones at 25,000 time step. At the beginning of sintering, particles approach to each other under attractive sintering forces with small relative velocities. According to the first term of the right hand side in Eq. (1a), a small approaching velocity leads to a small repulsive force, which leads to an attractive resultant force (compressive). With the sintering time going, the approaching velocities between contact particles increase, which results in an increasing repulsive force and then the resultant force will become repulsive.

Fig. $4 \mathrm{c}$ and d give the snapshots of force networks at 517,420 and 1785,000 time steps, respectively, where we can find tangled chains with compressive and tensile forces at a large sintering time step. The tangled structure consists of four layers of force chains, which are labeled as $L 1-L 4$ shown in Fig. $4 \mathrm{c}$. The forces on the first and third layers are compressive (red lines) while those on the second and fourth layers are tensile (black lines). In most cases, such a layer structure consists of two layers with opposite forces as shown at the right upper side of Fig. $4 \mathrm{c}$ and $\mathrm{d}$. The force chains could go across almost the whole system as shown in Fig. 4d, where the force chains originate from the position of force concentration at the top of the system, and then extend in the system with a decreasing magnitude. Opposite forces would be induced in front of the spreading line. For example, the tensile force spreads from the right upper side to the lower left one in the system shown in Fig. 4d with a decreasing magnitude; compressive forces are induced in front of the tensile one as shown by an arrow in Fig. 4d. Details can be found from the inset in Fig. 4d, which is a magnification of the local force network. Particles 2 and 3 in the inset tend to separate from each other due to high repulsive forces (two black lines), which activates a high attractive force (the red line) between the two contact particles trying to resist the separation. Thus, multi-layer force structures are often found in a sintering system. Comparing Fig. 4a and b to Fig. 4c and d, the sintering forces distribute much more non-uniformly with an increasing sintering time.

It is interesting to find a special force structure at some local positions as shown in Fig. 5(a-d), which we call "force ring". Nine force rings are denoted as $R 1-R 9$ in Fig. $5 \mathrm{~d}$. The smallest rings, such as R1 and R5, consist of seven particles: one is in the ring and six on the ring boundary. The biggest ring includes seventeen particles, such as $R 4$. The contact forces between each pair of particles on the ring boundary have the same state (compressive or tensile), while the contact forces in the ring have an opposite state to those on the boundary. For example, contact forces on the ring boundaries of R1, R2, R5, R6, R7 and R8 are tensile, while those in the rings are all compressive. Contact forces on the ring boundaries of R3, R4 and R9 are compressive, while those in the rings are tensile. Such an interesting structure should be a special one of the tangled structure discussed above. Furthermore, all the contact 

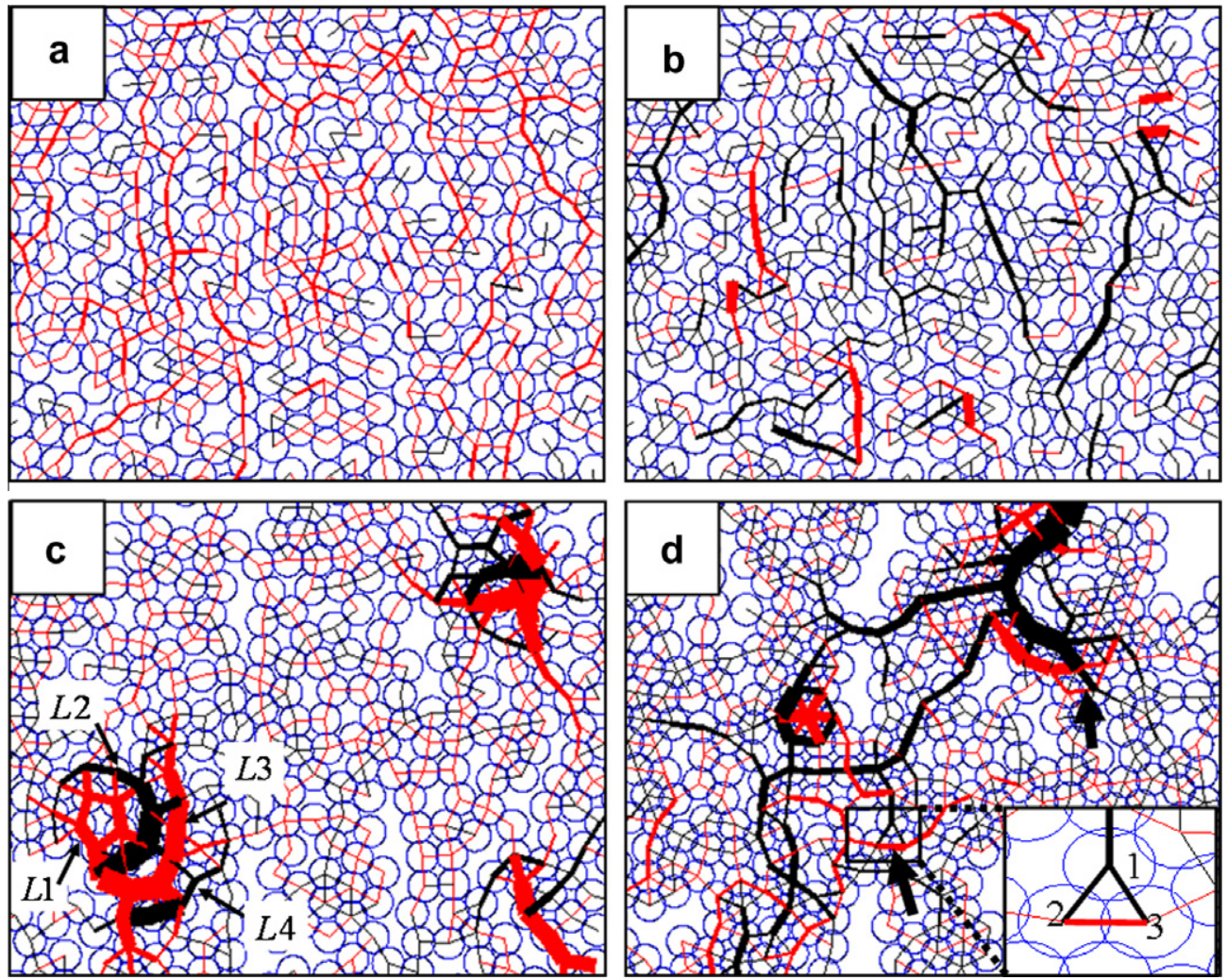

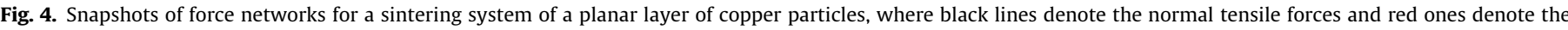

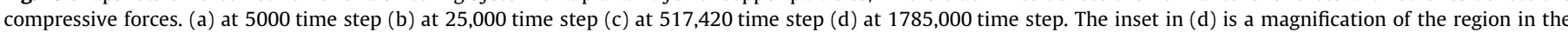
rectangle (For interpretation of the references to colour in this figure legend, the reader is referred to the web version of this article.)
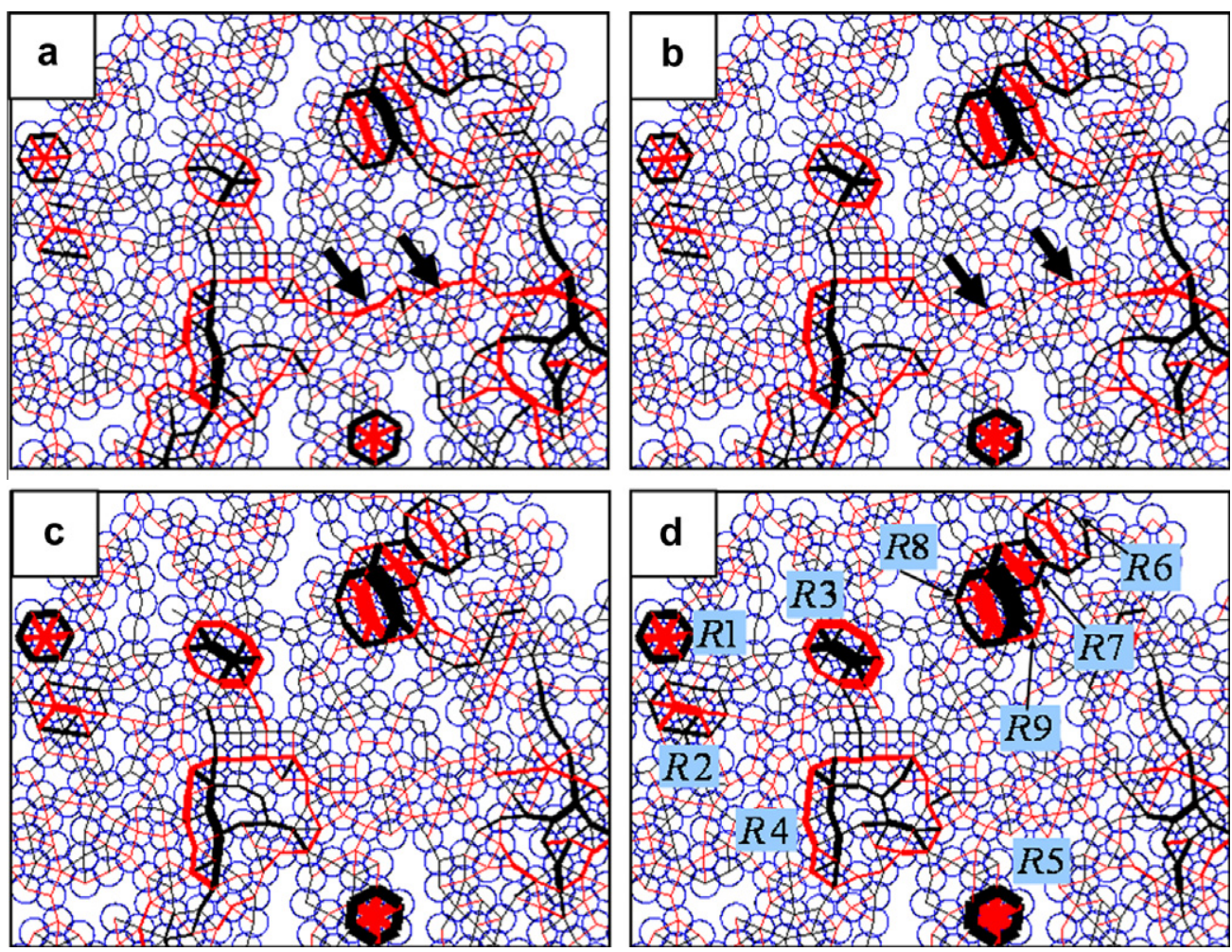

Fig. 5. Snapshots of the evolution of ring-like force chains. (a) at 1525,000 time step (b) at 1540,000 time step (c) at 1555,000 time step (d) at 1575,000 time step. 


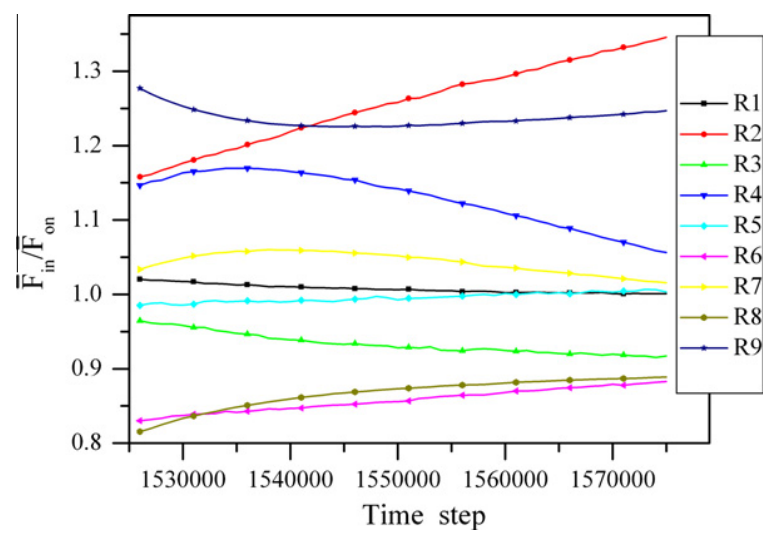

Fig. 6. Ratio of the average force $\bar{F}_{\text {in }}$ inside a ring to that $\bar{F}_{\text {on }}$ on the ring boundary as a function of the sintering time step, in which nine force rings labeled in Fig. $5 \mathrm{~d}$ are included.

forces inside a ring and on the ring boundary keep almost local equilibrium as depicted in Fig. 6 . The ratios of $\bar{F}_{\text {in }} / \bar{F}_{\text {on }}$ of nine force rings are almost close to 1 , especially for smaller rings $\mathrm{R} 1$ and $\mathrm{R} 5$, where $\bar{F}_{\text {in }}$ denotes the average magnitude of the contact forces inside a ring and $\bar{F}_{\text {on }}$ the average one of the contact forces on the ring boundary.
In addition, numerical simulations show that the High Force Chains (HFCs) always emerge suddenly, even the time step is very small. However, HFCs disappear gradually and a new HFC does not necessarily appear adjacent to another existing HFC. The new HFC is often activated far from the existing HFCs. Fig. 7a-d plot the snapshots of a new high force emerging at the upper-right corner of the system shown by an arrow, which is far from the existing HFCs at the left side of the system. Then, the new high force will induce other high forces to form a HFC as shown in Fig. 7c and d. The decay process of a HFC is shown in Fig. 8. The magnitude of a high force chain pointed by an arrow in Fig. 8a-c decreases gradually and finally disappears. After that, an opposite force arises at the same position in most cases but not all as shown in Fig. 8d-f, where the opposite force grows up little by little.

\subsection{Correlation between the contact network and the force network}

Numerical simulation shows that a high normal contact force is always activated at the site that a new contact forms. The results are given in Fig. 9 for a case of $\eta=0$ as an example. Same conclusions can be made for cases of $\eta \neq 0$. Fig. 9 gives a statistical result of the number of new forming contacts and the number of new contacts sustaining high forces at different simulation time step, where it is shown that both numbers coincide well with each other at almost all the time steps. There are 175 new forming contacts
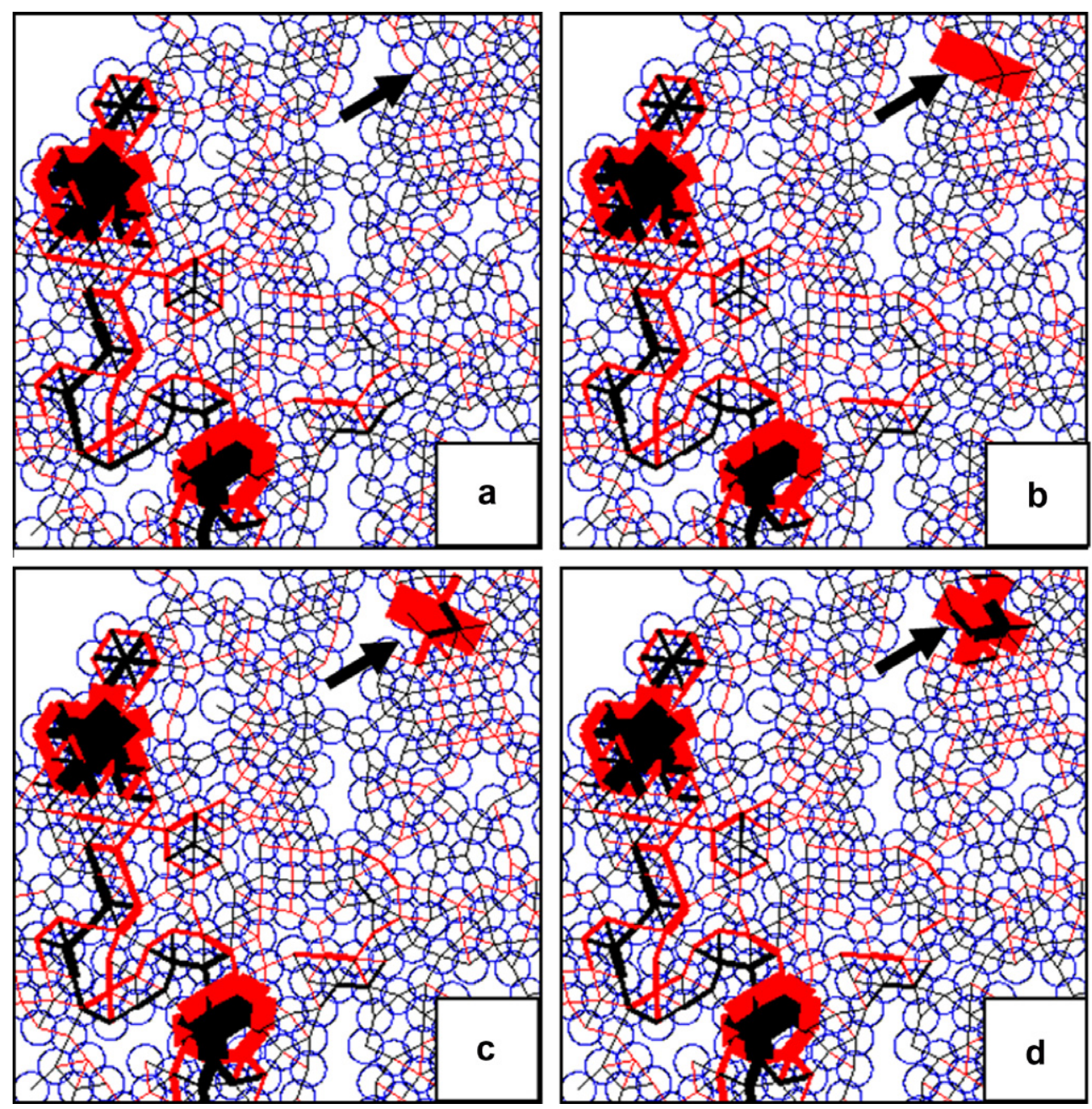

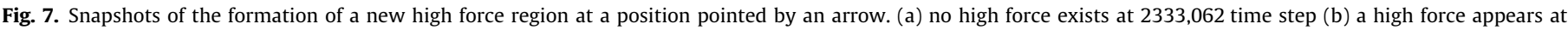
2333,063 time step ( $c$ and d) other high forces near the position propagate at 2333,066 and 2333,073 time step, respectively. 

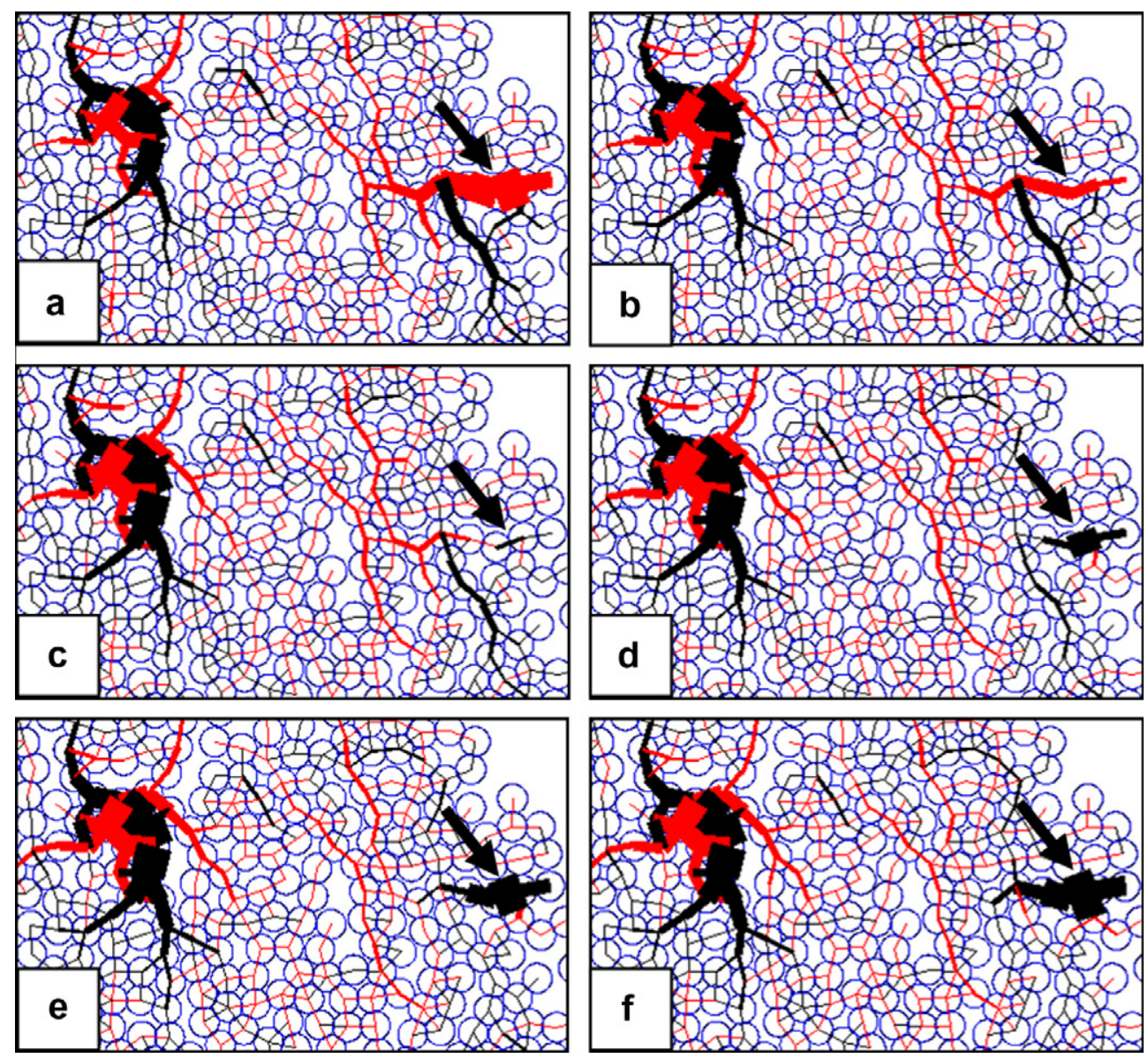

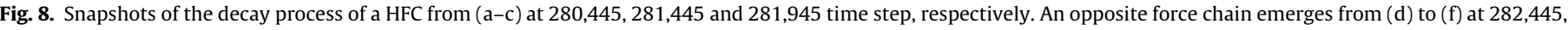
282,945 and 283,445 time step, respectively.

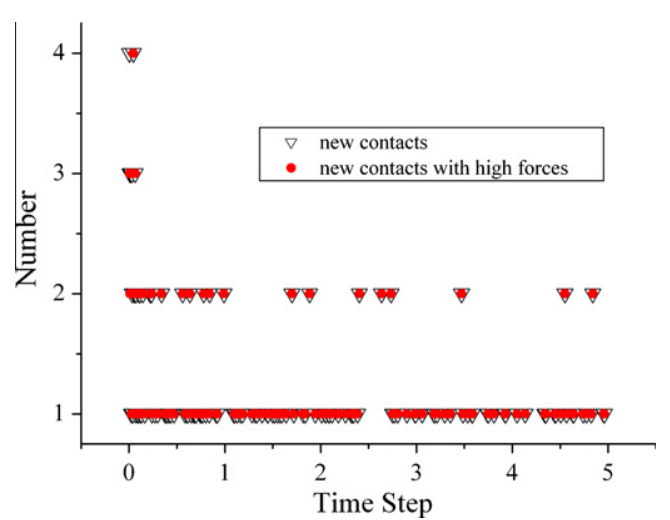

Fig. 9. Comparison between the number of new forming contacts and the number of new forming contacts sustaining high contact forces at different simulation time step.

totally, in which 172 of them sustain high normal contact forces. 100 of them sustain repulsive forces and the other 72 contacts sustain attractive ones. For a new contact, the normal contact force is not easy to achieve equilibrium due to the competition of the repulsively viscous force and the attractive sintering force as expressed in Eq. (1). Generally, it needs some time to balance the contact force at the site of a new forming contact. Any other correlations between the contact network and force network will be investigated in our future work, as well as the relation of the microstructure evolution and the force network, especially the force ring structure.

\section{Conclusions}

The microstructure evolution and force distribution have been studied by DEM as well as the complex network method at the particle length scale in solid-state sintering system of a planar layer of copper particles. We find that the evolution of microstructures is sensitive to the shape and size of contact networks. Pores tend to form in a large contact polygon and those existing in a small closed contact polygon will shrink and disappear finally. The distribution of the normal contact force is significantly non-uniform and the high force regions vary with the sintering time. Ring-like chains have been observed in the normal force networks, which can sustain longer time than other kinds of force chains.

Comparing the contact and force networks leads to a conclusion that a high normal contact force is always activated at the site of a new forming contact. However, it is far from enough to clarify all the correlations between the contact network and force network in a particle-sintering system. A number of significant problems are still open. For example, what is the effect of high forces on the evolution of contact networks? What is the relation between the force network and the microstructure evolution? How can we predict the microstructure evolution from the force network? For a sintering system with non-uniform particles, all these 
questions exist too. This work is just a beginning for us to use DEM as well as complex network method to understand the complex sintering process. Many important problems that will be helpful for understanding sintering systems and the fabrication of sintering products will be investigated in our future work.

\section{Acknowledgements}

This work is supported by the National Science Foundation of China (Nos. 10972220, 11125211 and 11021262) and the Nano-973 Project (2012CB937500).

\section{References}

[1] R.M. German, Sintering theory and practice, Wiley-Interscience, New York 1996.

[2] D. Bouvard, R.M. McMeeking, J. Am. Ceram. Soc. 79 (1996) 666.

[3] H.E. Exner, C. Muller, J. Am. Ceram. Soc. 92 (2009) 1384.

[4] G. Petzow, H.E. Exner, Z Metallk 67 (1976) 611.

[5] P.A. Cundall, O.D.L. Strack, Geotechnique 29 (1979) 47.

[6] E.A. Olevsky, Mater. Sci. Eng. R23 (1998) 60

[7] K. Rajendra, R.Z. Bordia, Acta Mater. 54 (2006) 8

[8] B. Henrich, A. Wonisch, T. Kraft, M. Moseler, H. Riedel, Acta Mater. 55 (2007) 753.

[9] C.L. Martin, LC.R. Schneider, L. Olmos, D. Bouvard, Scr. Mater. 55 (2006) 425

[10] C.L. Martin, D. Bouvard, G. Delette, J. Am. Ceram. Soc. 89 (2006) 3379.

[11] J.C. Kim, K.H. Auh, D.M. Martin, Model. Simul. Mater. Sci. Eng. 8 (2000) 159.

[12] L. Olmos, C.L. Martin, D. Bouvard, Powder Technol. 190 (2009) 134.
[13] F. Parhami, R.M. McMeeking, Mech. Mater. 27 (1998) 111.

[14] A. Wonisch, O. Guillon, T. Kraft, M. Moseler, H. Riedel, J. Rodel, Acta Mater. 55 (2007) 5187.

[15] C.L. Martin, R.K. Bordia, Acta Mater. 57 (2009) 549.

[16] C.L. Martin, H. Camacho-Montes, L. Olmos, D. Bouvard, R.K. Bordiaz, J. Am. Ceram. Soc. 92 (2009) 1435.

[17] L.F. Costa, O.N. Oliveira Jr., G. Travieso, F.A. Rodrigues, P.R.V. Boas, L. Antiqueira, M.P. Viana, L.E.C. Da Rocha, Adv. Phys. 60 (2011) 329.

[18] A. Arenas, A. Diaz-Guilera, J. Kurths, Y. Moreno, C.S. Zhou, Phys. Rep-Rev. Sec. Phys. Lett. 469 (2008) 93.

[19] S. Boccaletti, V. Latora, Y. Moreno, M. Chavez, D.U. Hwang, Phys. Rep-Rev. Sec. Phys. Lett. 424 (2006) 175.

[20] L.F. Costa, F.A. Rodrigues, G. Travieso, P.R.V. Boas, Adv. Phys. 56 (2007) 167.

[21] T.S. Evans, Contemp. Phys. 45 (2004) 455.

[22] F. Radjai, D.E. Wolf, M. Jean, J.J. Moreau, Phys. Rev. Lett. 80 (1998) 61

[23] F. Radjai, S. Roux, J.J. Moreau, Chaos 9 (1999) 544

[24] C.H. Liu, S.R. Nagel, D.A. Schecter, S.N. Coppersmith, S. Majumdar, O. Narayan, T.A. Witten, Science 269 (1995) 513.

[25] S. Ostojic, E. Somfai, B. Nienhuis, Nature 439 (2006) 828.

[26] L.E. Silbert, Phys. Rev. Lett. 94 (2005).

[27] L.E. Silbert, R.S. Farr, J.R. Melrose, R.C. Ball, J. Chem. Phys. 111 (1999) 4780.

[28] B.P. Tighe, J.H. Snoeijer, T.J.H. Vlugt, M. van Hecke, Soft Matter. 6 (2010) 2908.

[29] A. Tordesillas, P. O’Sullivan, D.M. Walker, Paramitha, C.R. Mec. 338 (2010) 556.

[30] A. Tordesillas, D.M. Walker, Q. Lin, Phys. Rev. E 81 (2010).

[31] C. Voivret, F. Radjai, J.Y. Delenne, M.S. El Youssoufi, Phys. Rev. Lett. (2009) 102.

[32] D.M. Walker, A. Tordesillas, Int. J. Solid. Struct. 47 (2010) 624.

[33] R.L. Coble, J. Appl. Phys. 32 (1961) 787.

[34] J.Z. Pan, A. Cocks, J. Rodel, R.Y. Huang, H.N. Ch'ng, J. Am. Ceram. Soc. 92 (2009) 1414.

[35] S. Coleman, W. Beere, Philos. Mag. 31 (1975) 1403.

[36] A. Wonisch, T. Kraft, M. Moseler, H. Riedel, J. Am. Ceram. Soc. 92 (2009) 1428. 